Journal of the Mathematical Society of Japan Vol. 5, No. 1 April, 1953.

\title{
On a direct transcendental singularity of an inverse function of a meromorphic function.
}

\author{
By Masatsugu TsujI
}

(Received Nov. 21, 1952)

Let $\Delta$ be an infinite domain on the $z$.plane, which may be infinitely multiply connected and $I^{\prime}$ be its boundary, which consists of at most a countable number of analytic curves. We assume that $I^{\prime}$ contains at least one curve extending to infinity. Let $w=w(z)$ be regular in $\Delta$ and on $I^{\prime}$, except at $z=\infty$, such that $|w(z)|<R$ in $\Delta$ and $|w(z)|=R$ on $\Gamma$ and $w(z) \neq 0$ in $\Delta$. Let $\Delta_{r}$ be the part of $\Delta$, which lies in $|z|<r$. We put

$$
\begin{gathered}
S(r ; \Delta)=\frac{1}{\pi} \iint_{\Delta_{r}} \frac{\left|w^{\prime}\right|^{2}}{\left(1+|w|^{2}\right)^{2}} d x d y,(w=w(z), z=x+i y), \\
T(r ; \Delta)=\int_{1}^{r} \underset{r}{S(r ; \Delta)} d r .
\end{gathered}
$$

Now $\Delta_{r}$ consists of a finite number of connected domains. Let $\Delta_{r}^{0}$ $\left(r \geqq r_{0}\right)$ be the one, which contains a fixed point $z_{0}$ of $\Delta$ and $\theta_{r}$ be the part of $|z|=r$, which belongs to the boundary of $\Delta_{r}^{0}$. $\theta_{r}$ consists of a finite number of arcs $\theta_{r}^{i}(i=1,2, \cdots, \nu(r))$ and $r \theta_{i}(r)$ be its arc length and put $\theta(r)=\sum \theta_{i}(r) . \quad \theta(r)$ is continuous except at most a countable number of isolated points $0<r_{1}<r_{2}<\cdots<r_{\nu} \rightarrow \infty$, where $\theta\left(r_{\nu}-0\right)$ $=\theta\left(r_{\nu}\right)<\theta\left(r_{\nu}+0\right)$.

In the former paper," I have proved the following theorem.

THEOREM. For any $0<\alpha<1$,

$$
T(r ; \Delta) \geqq \text { const. } e^{\pi \int_{r_{0}}^{\alpha r} \frac{d r}{r(r)}}\left(r \geqq r_{0}\right) \text {. }
$$

1) M. Tsuji: On a regular function which is of constant absolute value on the boundary of an infinite domain. Tohoku Math. Journ. 3 (1951). 
In the proof of the theorem on the number of direct trans. cendental singularities of an inverse function of a meromorphic function of finite order, Ahlfors ${ }^{2)}$ proved a similar relation :

$$
T(r) \geq \text { const. } e^{\pi \int_{r_{0}}^{a r} \underset{r(r)}{d r},}
$$

where $w(z)$ is meromorphic for $|z|<\infty$ and $T(r)$ is its characteristic function and $\theta(r)$ is defined for a simply connected domain, which is bounded by the outermost boundary curve of $\Delta$. Our theorem is an extension of (3). In this paper, I shall give a somewhat simpler proof than the former one.

Proof. Let

$$
u(z)=\log \begin{gathered}
R^{2}+|w|^{2} \\
2 R|w|
\end{gathered} \geqq 0, \quad w=w(z),
$$

then

$$
\begin{array}{r}
\Delta u=\frac{\partial^{2} u}{\partial y^{2}}+\frac{\partial^{2} u}{\partial y^{2}}=\begin{array}{r}
4 R^{2}\left|w^{\prime}\right|^{2} \\
\left(R^{2}+|w|^{2}\right)^{2}
\end{array} \geqq 0, \begin{array}{r}
\partial^{2} u \\
\partial \log r^{2}
\end{array}+\frac{\partial^{2} u}{\partial \theta^{2}}=r^{2} \Delta u \geqq 0, \\
\left(z=x+i y=r e^{i \theta}\right)
\end{array}
$$

so that $u(z)$ is subharmonic in $\Delta$.

Since $\begin{gathered}R^{2}+|w|^{2} \\ 2 R|w|\end{gathered}=1+\frac{(R-|w|)^{2}}{2 R|w|}$, we see that

$$
u=0, \frac{\partial u}{\partial \nu}=0 \text { on } I^{\prime},
$$

where $\nu$ is the outer normal of $I^{\prime}$.

Let $\lambda_{r}$ be the part of $|z|=r$, which lies in $\Delta$, so that $\theta_{r} \subset \lambda_{r}$. We put

$$
\mu(r)=\int_{\lambda_{r}} u\left(r e^{i \theta}\right) d \theta .
$$

We denote $I_{r}$ the part of $I^{r}$, which belongs to the boundary of $\Delta_{r}$, so that $I_{r}+\lambda_{r}$ is the whole boundary of $\Delta_{r}$.

2) L. Ahlfors: Über die asymptotischen Werte der meromorphen Funktionen endlicher Ordnung. Acta Acad. Aboensis. Math. et Phys. 6, Nr. 9 (1932). 
Since $u=0$ at the end points of $\lambda$. we have by (6) and Green's formula,

$$
\begin{aligned}
& r \mu^{\prime}(r)=\int_{\lambda_{r} \partial r} \partial u d \theta=\int_{{ }_{r}+\lambda_{r} \partial \nu^{\prime}} \partial u d s:=\iint_{t_{r}} \Delta u d x d y= \\
& 4 R^{\prime} \iint_{A_{r},} \frac{\left.\left|2 w^{\prime}\right|^{2}+|2|^{2}\right)^{2}}{d x d y>0}
\end{aligned}
$$

so that

$$
\begin{aligned}
& \int_{\lambda_{r}} u\left(r c^{i \theta}\right) d \theta=-\mu(r)=4 R^{2} \int_{r_{0}}^{r} d r \iint_{J_{r}\left(R^{\prime \prime}+\left|w^{2}\right|^{2}\right.} d x d y+\text { const. } \leq \\
& \text { const. } \int_{r, 0}^{r} d r \iint_{\Delta_{r}\left(1+|u|^{2}\right)^{2}} d x d y+\text { const. }=\text { const. } T(r ; d)+\text { const. }
\end{aligned}
$$

Following Carleman's', we put

$$
\left.m(r)=\int_{2 \pi}^{1} \int_{0,}\left[u\left(r^{i \theta}\right)\right]^{\prime} d t\right)
$$

We denote $I_{r}^{r 0}$ the part of $I^{\prime}$, which belongs to the boundary of $A_{r}^{0}$, so that $I_{r}^{r 0}+\theta_{r}$ is the whole boundary of $\mathcal{S}_{r}^{0}$. Since $u=0$ at the end points of $\theta_{r}$ by (6) and Green's formula, we have for $r_{\nu}<r<r_{2}, 1$,

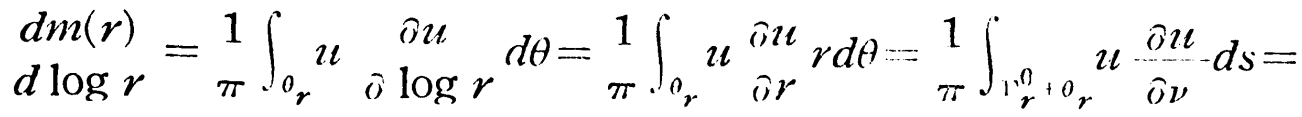

$$
\begin{aligned}
& 1 \iint_{\Delta_{r}^{0}}\left(\left(\frac{\partial u}{\partial x}\right)^{2}+\left(\frac{\partial u}{\partial y}\right)^{2}\right) d x d y+\frac{1}{\pi} \iint_{J_{r}^{0}} u \Delta u d x d y>0 \text {, }
\end{aligned}
$$

so that $m(r)$ increases at $r\left(\neq r_{\nu}\right)$. At $r_{\nu}$, we see that $m\left(r_{\nu}-0\right)=m\left(r_{\nu}\right)$ $<m\left(r_{\nu}+0\right)$. Hence $m(r)$ is an increasing function of $r$.

By (5), we have

$$
\begin{aligned}
& \begin{array}{l}
d^{2} m(r) \\
d \log r^{2}
\end{array}=\frac{1}{\pi} \int_{\sigma_{r}}\left(\left(\begin{array}{c}
\partial u \\
\partial \log r
\end{array}\right)^{2}+u \begin{array}{c}
\partial^{2} u \\
\partial \log r^{2}
\end{array}\right) d \theta= \\
& 1 \int_{\pi}\left(\left(\frac{\partial u}{\partial \log r}\right)^{2}+u r^{2} \Delta u-u\left(\begin{array}{c}
\partial \theta^{2} \\
\partial \theta^{2}
\end{array}\right) d \theta>\right.
\end{aligned}
$$

3) T. Carleman: Sur une inegalité différentielle dans la théorie des fonctions analytiques. C. R. 196 (1936). 
78

M. TsujI

$$
\frac{1}{\pi} \int_{\theta_{r}}\left(\left(\frac{\partial u}{\partial \log r}\right)^{2}-u \frac{\partial^{2} u}{\partial \theta^{2}}\right) d \theta=\frac{1}{\pi} \int_{\theta_{r}}\left(\left(\frac{\partial u}{\partial \log r}\right)^{2}+\left(\frac{\partial u}{\partial \theta}\right)^{2}\right) d \theta,
$$

by the integration by parts.

Now

$$
\left(\frac{d m(r)}{d \log r}\right)^{2} \leqq \frac{1}{\pi^{2}} \int_{\theta_{r}} u^{2} d \theta \int_{\theta_{r}}\left(\frac{\partial u}{\partial \log r}\right)^{2} d \theta=\frac{2 m(r)}{\pi} \int_{\theta_{r}}\left(\frac{\partial u}{\partial \log r}\right)^{2} d \theta,
$$

so that

$$
\frac{1}{\pi} \int_{\theta_{r}}\left(\frac{\partial u}{\partial \log r}\right)^{2} d \theta \geqq \frac{1}{2 m(r)}\left(\frac{d m(r)}{d \log r}\right)^{2}
$$

Since $u=0$ at the end pints of $\theta_{r}^{i}$, we have by Wirtinger's inequality,

$$
\int_{\theta_{r}^{i}}\left(\frac{\partial u}{\partial \theta}\right)^{2} d \theta \geqq \frac{\pi^{2}}{\left(\theta_{i}(r)\right)^{2}} \int_{\theta_{r}^{i}} u^{2} d \theta \geqq \frac{\pi^{2}}{(\theta(r))^{2}} \int_{\theta_{r}^{i}} u^{2} d \theta
$$

Summing up for $i$,

$$
\frac{1}{\pi} \int_{\theta_{r}}\left(\frac{\partial u}{\partial \theta}\right)^{2} d \theta \geqq \frac{\pi}{(\theta(r))^{2}} \int_{\theta_{r}} u^{2} d \theta=\frac{1}{2}\left(\frac{2 \pi}{\theta(r)}\right)^{2} m(r) \text {. }
$$

Hence by (12), (13), (14),

$$
\begin{gathered}
\frac{d^{2} m(r)}{d \log r^{2}} \geqq \frac{1}{2 m(r)}\left(\frac{d m(r)}{d \log r}\right)^{2}+\frac{1}{2}\left(\frac{2 \pi}{\theta(r)}\right)^{2} m(r) \\
\geqq \frac{d m(r)}{d \log r} \cdot \frac{2 \pi}{\theta(r)}\left(r_{\nu}<r<r_{\nu+1}\right),
\end{gathered}
$$

since $\frac{d m(r)}{d \log r}>0$ by (11).

From (11), we see that at $r_{\nu}$,

$$
\left(\frac{d m(r)}{d \log r}\right)_{r_{\nu}-0}=\left(\frac{d m(r)}{d \log r}\right)_{r_{\nu}}<\left(\frac{d m(r)}{d \log r}\right)_{r_{\nu}+0} .
$$

so that integrating (15), we have

$$
\log \frac{d m(r)}{d \log r}-\log \frac{d m\left(r_{0}\right)}{d \log r_{0}} \geqq 2 \pi \int_{r_{0}}^{r} \frac{d r}{r \theta(r)},
$$


or

$$
r m^{\prime}(r)=\begin{aligned}
& d m(r) \\
& d \log r
\end{aligned} \geq \text { const. } e^{2 \pi \int_{r_{0}}^{r}-d r}
$$

Since $m(r)$ is an increasing function of $r$, we have for any $0<\beta<1$,

$$
\begin{aligned}
& m(r)>m(r)-m\left(r_{0}\right) \geqq \int_{r_{0}}^{r} m^{\prime}(r) d r \geqq \text { const. } \int_{r_{r}}^{r} d r e^{\left.2 \pi\right|_{r} ^{r}} \int_{r(t)}^{d t} \geq
\end{aligned}
$$

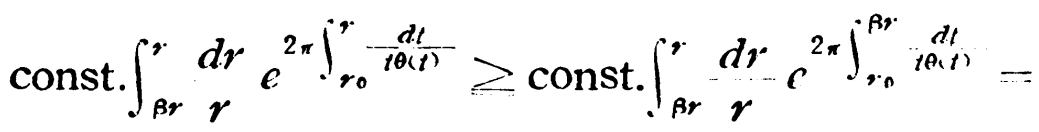

$$
\begin{aligned}
& \text { const. } e^{2 \pi \int_{r \cdot r \cdot r \cdot r}^{\beta r}}
\end{aligned}
$$

Let $u(z)$ attain its maximum at $z=r c^{i \theta_{0}}$ on $\theta_{r}$, then

$$
\left(u\left(r e^{i \theta_{0}}\right)\right)^{2} \geq \frac{1}{2 \pi} \int_{\theta_{r}}\left[\left.u\left(r c^{i \theta}\right)\right|^{2} d \theta=m(r) \geq \text { const. } c^{2 \pi \int_{, r}^{\beta r} \underset{r(r)}{d r},},\right.
$$

so that

$$
u\left(r e^{i \theta_{n}}\right) \geq \text { const. } e^{\pi)_{r_{0}}^{\beta r} \cdot r_{r(r)}^{d r}}
$$

Let

$$
\begin{array}{rl}
U(z)=U\left(r e^{i \theta}\right)=\frac{1}{2 \pi} \int_{\lambda_{\rho}} u\left(\rho e^{i \varphi}\right) & \rho^{2}-r^{2} \\
\rho^{2}-2 \rho r \cos (\phi-\theta)+r^{2} & d \phi, \\
& (r<\rho)
\end{array}
$$

then $U(z)=u(z)$ on $\lambda_{\rho}$ and $U(z)>0=u(z)$ on $\Gamma_{p}$ and since $u(z)$ is subharmonic in $\Delta_{\rho}$, we have $u(z)<U(z)$ in $\Delta_{\mathfrak{p}}$. Hence if we put $\rho=k r(k>1)$, then by (19) and (9),

$$
\begin{aligned}
& u\left(r e^{i \theta_{0}}\right)<U\left(r e^{i \theta_{0}}\right) \leqq \underset{\rho-r}{\rho+r} \cdot \frac{1}{2 \pi} \int_{\lambda_{\rho}} u\left(\rho \rho^{i \varphi}\right) d \phi=\begin{array}{l}
k+1 \\
k-1
\end{array} \cdot \frac{1}{2 \pi} \int_{\lambda_{k r}} u\left(k r e^{i \varphi}\right) d \phi \\
& \leqq \text { const. } T(k r ; \Delta)+\text { const., }
\end{aligned}
$$


so that by (18),

$$
T(k r ; \Delta) \geq \text { const. } e^{\pi \int_{r_{0}}^{\beta r} \frac{d r}{r \theta(r)}}-\text { const. }
$$

Hence if we put $r$ instead of $k r$ and $\alpha=\frac{\beta}{k}$, then

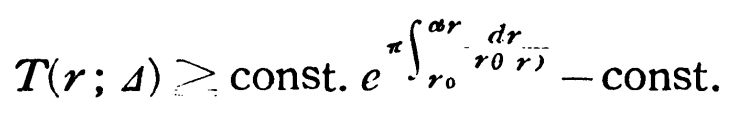

From this we have easily,

$$
T(r ; \Delta) \geqq \text { const. } e^{\pi \int_{r_{0}}^{\alpha r} r(r)}\left(r \geqslant r_{0}\right) .
$$

Since $\alpha$ is any number, such that $0<\alpha<1$, our theorem is proved.

Mathematical Institute, Tokyo University. 